\title{
Pediatric Liver Biopsy: A Clinicopathologic Study
}

\author{
Pragati Aditya Sathe* and Dipali Eknath Mahale \\ Department of Pathology, Seth GS Medical College\& KEM Hospital, Mumbai, India
}

\section{ABSTRACT}

Introduction: The spectrum of liver diseases in children differs from that in adults. Different diagnostic modalities which are available are not capable of diagnosing the liver disease individually unless they are supplemented by a liver biopsy. Hence liver biopsy is an invaluable tool in the diagnosis of liver diseases.

Aims and Objectives: The aims of this study were to study the spectrum of liver diseases in pediatric age group at our institute, to correlate the liver biopsy findings with clinical findings and laboratory investigations and to review the literature on this topic.

Results: We received a total of 130 liver biopsies during the five year study. Out of these, 65 biopsies were performed in children below one year of age while the remaining 65 liver biopsies belonged to children aged more than one year. The common indications for performing liver biopsies included prolonged jaundice, clay colored stools, abdominal distension, abdominal pain, suspected inborn error of metabolism and for a suspected hepatic tumor. Extrahepatic biliary atresia was the commonest pathology found in children below one year of age whereas metabolic disease was the most common pathology found in children above one year of age. Special stains helped in refining the diagnosis further. Laboratory investigations were correlated to offer a complete diagnosis.

Conclusion: Paediatric liver biopsy is of value in diagnosis of disorders where clinical and laboratory parameters overlap. At the same time, clinical and laboratory correlation is a must due to overlapping histologic features of many liver diseases.

Keywords: Pediatric, Liver, Biopsy, Metabolic Diseases, Cholestasis

\section{Introduction}

The spectrum of pediatric liver diseases is different than in adults. In pediatric age group, liver suffers from metabolic, infective, cholestatic and neoplastic disorders resulting in abnormal liver function tests, jaundice and hepatomegaly. ${ }^{[1]}$ Various diagnostic modalities are available such as liver function tests, serum tumor markers and radiologic imaging techniques like ultrasonography (USG), computed tomography (CT) and Hydroxy iminodiacetic acid (HIDA) scan.$^{[1,2]}$ All these cannot individually suggest an accurate diagnosis in liver diseases. Liver biopsy, though invasive, is therefore an important diagnostic tool for liver diseases. ${ }^{[1]}$

\section{Materials and Methods}

This is retrospective analysis of liver biopsies performed in patients below 12 years of age received at our department over a five year period.

Clinical features, laboratory investigations (liver function tests, serum copper levels, serological markers for viral infections, tumor markers), radiologic findings (USG, CT, HIDA scan) and histologic findings (including the findings on routine as well as special stains) were noted from the hospital and department records of patients. Further clinicopathologic correlation and comparison with similar studies in literature was done.

\section{Results}

A total of 130 liver biopsies belonging to paediatric age group (less than 12 years) were received in the department of pathology during the study period comprising $0.23 \%$ of total surgical workload $(55,020$ specimens $)$ and $4.3 \%$ of pediatric surgical workload (3010 specimens) received in the surgical pathology laboratory during this period.

Table 1 summarizes the spectrum of the liver lesions received according to their frequency. As the spectrum of liver diseases differs between infants and children more than one year old, the cases have been categorised into two groups. We received equal number of cases in both groups (65 cases in each).The most common liver disease affecting infants was EHBA (38.46\%) followed by neonatal hepatitis (24.61\%). Metabolic disorders (12.3\%) were commonest liver pathology seen in children aged more than 1 year.

Extrahepatic Biliary Atresia: (Fig 1 a, b) - A total of 26 cases of EHBA were found in our study. Among these, 25 were infants. The commonest symptom was icterus followed by clay colored stools. Liver function test results were available in 17 cases. Liver enzymes were elevated in the following range -SGOT (131-405 U/L), SGPT (51- 
$158 \mathrm{U} / \mathrm{L})$, and Total bilirubin (2.6-15 mg/dl), D.bilirubin (1.6-9.6 mg/dl).HIDA and USG were available in all cases. In 24 cases, radiology was diagnostic of EHBA. In three cases, a differential diagnosis of neonatal hepatitis was given. Features overlapping with $\mathrm{NH}$ were seen in one case which was also positive for cytomegalovirus (CMV) Immunoglobulin $\mathrm{G}(\mathrm{IgG})$. Hence a differential diagnosis was given in this case. All patients except one underwent Kasai's procedure.

Neonatal Hepatitis: (Fig 1 c, d) - A total of 18 cases of neonatal hepatitis were found in our study. Among these, 17 were infants and one case was a child aged 2.5 years. The most common symptom was icterus followed by abdominal distension.Liver function test results were available in 11 cases. Liver enzymes were elevated in the following range -SGOT (100-724 U/L), SGPT (66-367 U/L), Total bilirubin (4-22 mg/dl), Direct bilirubin (3.2-17.2mg/dl).Four cases showed raised titres for CMV. TORCH titre was done in one case which was negative. Test for Alpha -1- antitrypsin deficiency was done in one case which was negative.

Cirrhosis: A total of eight cases of cirrhosis without identifiable etiology were found in our study. All eight cases were children aged above one year of age.The most common symptom was abdominal distension. Liver function test results were available in seven cases. Liver enzymes were elevated in the following range -SGOT (60$181 \mathrm{U} / \mathrm{L})$, SGPT (68-129 U/L), total bilirubin (9-19 mg/dl), direct bilirubin (5-8.4 mg/dl).In one case, the cirrhosis was of micronodular type. Special stains such as PAS, Orcein and Prussian blue were negative.

Glycogen Storage Disease: A total of 12 cases of glycogen storage diseases were found in our study (Figure 2e). Among these, only two patients were infants and the remaining nine cases were children aged above one year of age.Most common symptom was abdominal distension (11 cases). Others were delayed milestones (4 cases), dolls facies (1 case), hypotonia (1 case) and convulsions (2 cases). Five cases had progressed to cirrhosis. All 12 cases showed PAS positivity and diastase sensitivity indicating presence of glycogen. None of the cases had PAS positive diastase resistant globules that would have suggested Type IV disease.

Two cases showed the morphology of glycogen storage disease type III whereas in two cases there was overlap morphology of type 3 with type II and type I respectively. The remaining cases showed morphology of Type I disease. Confirmation was not possible as the enzyme studies were not available in our study.

Lipid Storage Disorder: was seen in two patients. Both cases belonged to the first group. Clinically they presented with abdominal distension, lethargy, vomiting and icterus. One patient also had developmental delay and puffy face. Liver function test ranged as follows - SGOT (92-450 U/1), SGPT (82-342U/L), total bilirubin (10.6-10mg/dl), direct bilirubin (4.8-5.48 mg/dl) Further workup was not available and hence further categorization was not possible.

Secondary Hemosiderosis: was seen in two patients, six and 11- year -old respectively (Figure $2 \mathrm{a}, \mathrm{b}$ ). Both patients suffered from Thalassemia major. SGOT and SGPT were raised in one patient. Both cases had progressed to cirrhosis.

Gaucher Disease: was noted in a four- month- old female child who presented with abdominal distension, fever and delayed milestones. Though the histology was characteristic, enzyme studies could not be performed.

Chronic Hepatitis: A total of 11 cases of chronic hepatitis were found in our study. Among these, only two patients were infants and the remaining nine cases were children aged above one year of age.

Most common symptom was abdominal distension. Liver function test results were available in six cases. Liver enzymes were elevated in the following range -SGOT (Normal-117 U/L), SGPT (Normal- $95 \mathrm{U} / \mathrm{L}$ ), total bilirubin (1.4-1.6 mg/dl) and direct bilirubin (0.6-0.8 mg/dl).

Five cases had progressed to cirrhosis. Grading and staging was done using Ishak scoring system. The histology showed features of grade 1 to 3 and stage 1 to 4 . Viral markers were negative in all cases. Reticulin stain showed features of incomplete cirrhosis in one case and cirrhosis in four cases.

Biliary Disorders: A total of 13 cases were found in our study in this group. Among these, nine patients were infants and the remaining four cases were children aged above one year of age. We had progressive intrahepatic cholestatic disorder ( 1 case) and two cases each of paucity of bile ductules, choledochal cyst and sclerosing cholangitis. Most common symptom was icterus followed by abdominal distension.

Liver function test results were available in seven cases. Liver enzymes were elevated in the following range -SGOT (100-209 U/L), SGPT (67-109 U/L), total bilirubin (Normal- $13.4 \mathrm{mg} / \mathrm{dl}$ ), and direct bilirubin (Normal- $9.2 \mathrm{mg} / \mathrm{dl}$ )

Orcein was positive for copper associated protein in the case of PFIC. Seven cases could not be classified into any particular disorder.

Wilson Disease: A total of three cases of Wilson disease were found in our study. Among these, all patients were above one year of age. 
The symptoms were abdominal distension, fever, drooling of saliva, ataxia and tremors. Kayser-Fleischer (KF) ring was present in three cases. Liver function test results were available in three cases. Liver enzymes were elevated in the following range -SGOT (Normal -233U/L), SGPT (Normal-134U/L). Total protein was normal. High urine copper level was found in two cases and low serum ceruloplasmin was found in one of these cases. Histology in all cases was that of chronic hepatitis with cirrhosis. Dry weight of copper could not be performed in any case.

Granulomatous Hepatitis: A total of four cases of granulomatous hepatitis were found in our study. Among these, two patients were infants and two cases were children above one year of age.

The symptoms were fever, abdominal distension and pain, icterus and delayed development. USG was available in three cases. Histology showed features of granulomatous hepatitis favoring tuberculosis etiology in three cases. There was associated vertebral tuberculosis in one case. One out of four cases did not have demonstrable etiology for granulomas.

Autoimmune Hepatitis: A total of two cases of autoimmune hepatitis were found in our study. Both cases were children above one year of age.

The symptoms were abdominal distension, icterus, fever and vomiting. Liver function test results were available in both cases. Liver enzymes were elevated in the following range-SGOT (234-300 U/L), SGPT (184-280 U/L), total bilirubin $(3.2-5 \mathrm{mg} / \mathrm{dl})$, direct bilirubin $(1.4-4 \mathrm{mg} / \mathrm{dl})$.
Antibody studies were available in one case and showed antinuclear antibody positive.

Congenital Hepatic Fibrosis (CHF): was seen in two cases (Figure 2c, 2d). Both were six year old and presented with abdominal distension. Total bilirubin, SGOT, SGPT were normal. USG showed hepatosplenomegaly, features of liver parenchymal disease and portal hypertension. There was no associated organ disease.

Budd Chiari Syndrome (BCS): was seen in two patients who presented with abdominal distension and right hypochondriac pain. SGOT, SGPT, total bilirubin, direct bilirubin were normal. CT abdomen showed liver cirrhosis. The diagnosis was based on clinical correlation.

Hepatic Tumors: A total of four cases of liver tumours were found in our study (Figure $2 \mathrm{f}$ ). Among these, only one case was an infant and three cases were children above one year of age.

The symptoms were abdominal distension, fever and abdominal pain.

AFP levels were available in three cases and showed normal to high value.

Histology in the four cases showed features of hepatoblastoma fetal type, Non Hodgkin's lymphoma (two cases), and high grade malignant tumour possibility of sarcoma (one case). The tumor cells were positive only for Vimentin in the last case confirming the diagnosis of sarcoma without further categorization.

Three liver biopsies did not fulfill criteria of adequacy on histology and three biopsies were autolysed.

Table 1: Age wise distribution of cases $(n=130)$.

\begin{tabular}{|l|c|c|c|c|}
\hline Diagnosis & $\begin{array}{c}\text { No. of cases } \\
<1 \text { year }(\mathbf{n}=\mathbf{6 5})\end{array}$ & $\begin{array}{c}\text { \% of total } \\
\text { cases }\end{array}$ & $\begin{array}{c}\text { No. of cases } \\
>1 \text { year }(\mathbf{n}=65)\end{array}$ & $\begin{array}{c}\text { \%of total } \\
\text { cases }\end{array}$ \\
\hline EHBA & 26 & $20 \%$ & 1 & $0.76 \%$ \\
\hline Neonatal hepatitis & 16 & $12.3 \%$ & 2 & $1.53 \%$ \\
\hline Metabolic disorders & 4 & & $\mathbf{1 6}$ & \\
Glycogen storage disorder & 2 & & 9 & \\
Wilson disease & 0 & $3.07 \%$ & 3 & $12.3 \%$ \\
Lipid metabolic disorder & 1 & & 2 & \\
Hemosiderosis & 0 & & 0 & \\
Gaucher Disease & 1 & & 1 & $10 \%$ \\
Carbohydrate Metabolic disorder & 0 & & 9 & \\
\hline Hepatitis & 4 & $3.07 \%$ & 2 & \\
Chronic hepatitis & 2 & & 2 & \\
Autoimmune hepatitis & 0 & & 2 & \\
Granulomatous hepatitis & 2 & & & \\
\hline
\end{tabular}




\begin{tabular}{|c|c|c|c|c|}
\hline Diagnosis & $\begin{array}{l}\text { No. of cases } \\
<1 \text { year }(n=65)\end{array}$ & $\begin{array}{l}\% \text { of total } \\
\text { cases }\end{array}$ & $\begin{array}{l}\text { No. of cases } \\
>1 \text { year }(n=65)\end{array}$ & $\begin{array}{l}\% \text { of total } \\
\text { cases }\end{array}$ \\
\hline Cirrhosis (No etiology demonstrated) & 0 & $0 \%$ & 8 & $6.15 \%$ \\
\hline \multicolumn{5}{|l|}{ Biliary diseases } \\
\hline Progressive familial intrahepatic & 9 & \multirow{7}{*}{$6.9 \%$} & 4 & \multirow{7}{*}{$3.07 \%$} \\
\hline cholestasis & 0 & & 1 & \\
\hline Paucity of bile ductules & & & & \\
\hline Choledochal cyst & 2 & & 0 & \\
\hline Sclerosing cholangitis & 2 & & 0 & \\
\hline Intrahepatic cholestatic disease due to & 0 & & 2 & \\
\hline $\begin{array}{l}\text { intrahepatic biliary disease } \\
\text { (Not classifiable) }\end{array}$ & 5 & & 2 & \\
\hline Malignancy & 1 & $0.76 \%$ & 3 & $2.30 \%$ \\
\hline Congenital Hepatic Fibrosis & 0 & $0 \%$ & 2 & $1.53 \%$ \\
\hline Budd Chiari syndrome & 0 & $0 \%$ & 2 & $1.53 \%$ \\
\hline Steatosis and steatohepatitis & 1 & $0.76 \%$ & 2 & $1.53 \%$ \\
\hline Hepatic necrosis (focal and diffuse) & 1 & $0.76 \%$ & 1 & $0.76 \%$ \\
\hline Non cirrhotic portal fibrosis & 0 & $0 \%$ & 1 & $0.76 \%$ \\
\hline Nonspecific portal inflammation & 0 & $0 \%$ & 3 & $2.30 \%$ \\
\hline No significant pathology & 0 & $0 \%$ & 4 & $3.07 \%$ \\
\hline Inadequate & 1 & $0.76 \%$ & 2 & $1.53 \%$ \\
\hline Autolysed & 2 & $1.53 \%$ & 1 & $0.76 \%$ \\
\hline Total & 65 & & 65 & \\
\hline
\end{tabular}

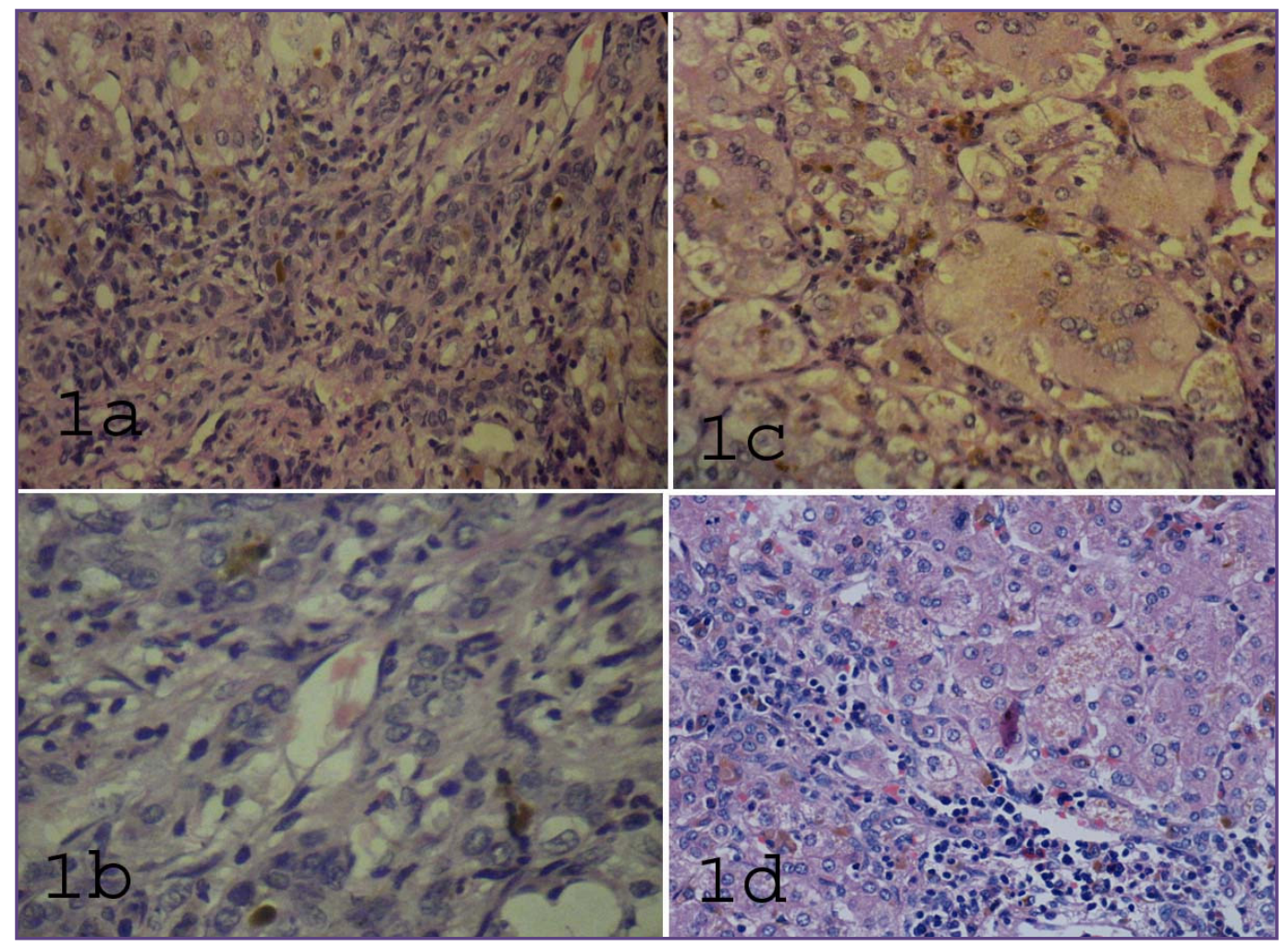

Fig 1: a. Extrahepatic biliary atresia: Proliferating bile ductules in the expanded portal tracts. (HE, $\mathbf{x} 400)$ b. Extrahepatic biliary atresia: Bile ductular plugging. (HE, x400) c. Neonatal hepatitis: Giant cell transformation. (HE, x400) d. Neonatal hepatitis: Inflammation and extramedullary hematopoiesis in the portal tracts. (HE, x 400). 

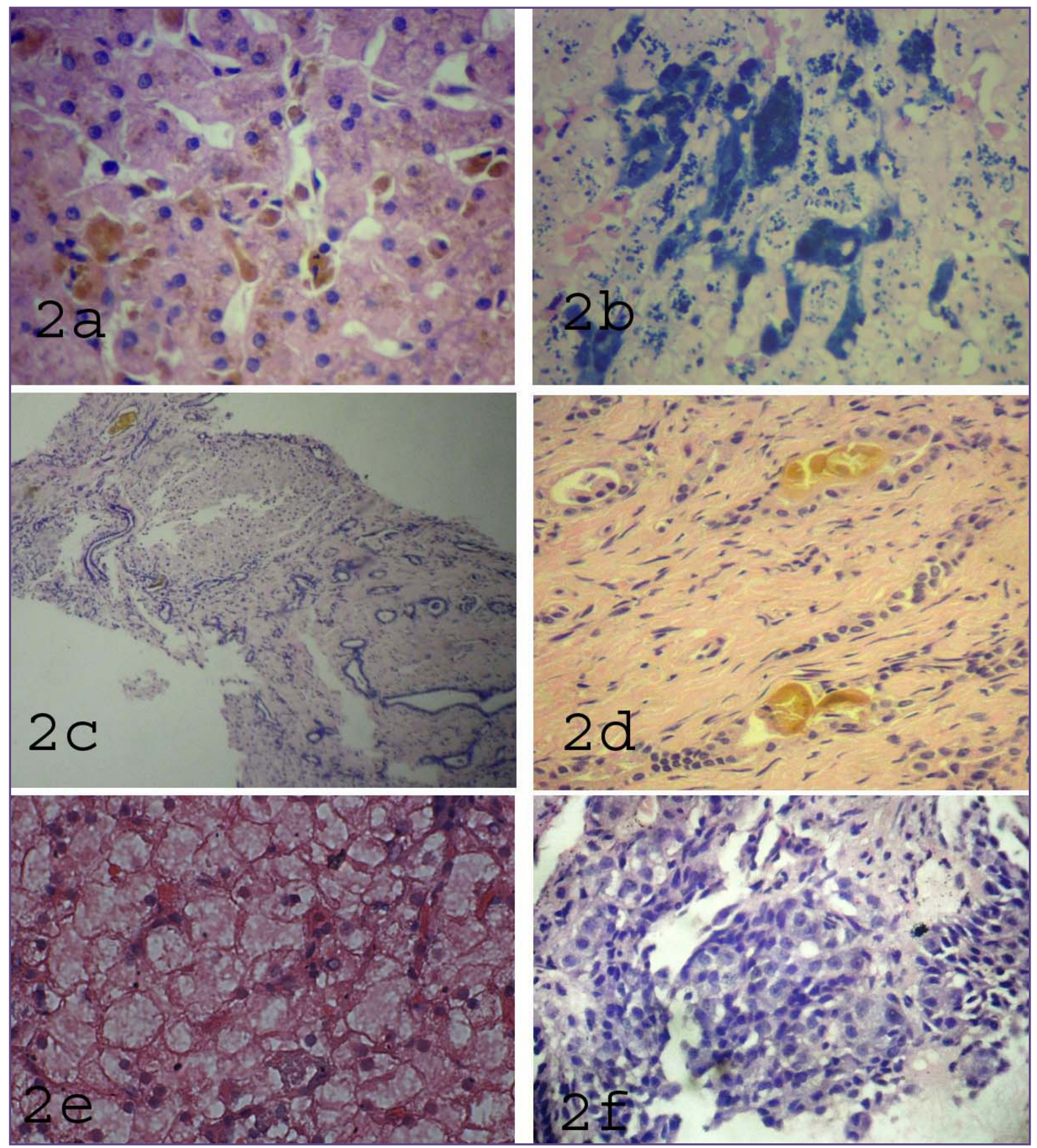

Fig. 2: a. Case of hemosiderosis showing brown pigment in the Kupffer cells. (HE, x 400) b. Prussian Blue stain highlighting the hemosiderin. (HE, x 100) c. Congenital hepatic fibrosis -Portal tracts show angulated bile ductules at the periphery of the tracts. (HE, $x$ 40) d. Bile plugging of the abnormal ductules. (HE, x 400) e. Glycogen storage disease - Distended pale hepatocytes. Few glycogenated nuclei are seen. (HE, x400) f. A case of hepatoblastoma - Cells show hyperchromatic nuclei and scant cytoplasm. Vague ductular arrangement was appreciated. (HE, x400). 


\section{Discussion}

The pattern of liver diseases in paediatric age group differs in infancy and in children beyond infancy. We found equal number of cases in children below and above one year of age. The common indications for performing liver biopsies included prolonged jaundice, clay colored stools, abdominal distension, abdominal pain, suspected inborn error of metabolism and for a suspected hepatic tumor. We also found a wide spectrum of liver diseases ranging from congenital to neoplastic diseases in both age groups. Cholestatic disease was found to be commonest in infancy. EHBA and neonatal hepatitis were the most common causes. Metabolic disorders, chronic hepatitis and cirrhosis were commonly seen in children aged more than one year. These findings were consistent with studies published by Ahmed $\mathrm{M}$ et al, Ramkrishna et al, and Muthuphei, et al. ${ }^{[1,3]}$ Some diseases like Reye's syndrome, fulminant hepatitis, schistosomiasis, echinococcus, hemophagocytic lymphohistiocytocytosis, and alpha-1-antitrypsin deficiency were not seen in our study.

The commonest clinical presentation of both $\mathrm{NH}$ and EHBA is icterus. EHBA in addition is associated with clay colored stools. ${ }^{[4}$ The clinical features and laboratory parameters and even histologic features in EHBA and $\mathrm{NH}$ can overlap..$^{[5}$ Presence of jaundice at birth, rise in alkaline phosphatase over other liver enzymes, HIDA scan and histologic features like proliferating biliary ductules with bile plugging is suggestive of EHBA (Figure $1 \mathrm{a}-\mathrm{d}) .{ }^{[6,7 .]}$ Frequently, portal ductal plate is sent to opine on the diameter of the largest bile ductule which helps in deciding the success of the surgical procedure in EHBA [5] The distinction between NH and EHBA is important as management differs. Neonatal hepatitis can be diagnosed as idiopathic only after etiology is not identified by serologic tests especially infections ${ }^{\left[{ }^{8]}\right.}$ Neonatal hepatitis can progress to cirrhosis rarely and was seen in two of our cases. Findings of periportal fibrosis, moderate to severe portal inflammation, and/or diffuse giant cell transformation appear to be major factors predictive for poor outcome in cases of neonatal hepatitis as concluded by Chang et al and were seen in three of our cases. ${ }^{[10]}$ Association with other congenital anomalies was reported in about one-fifth of cases by Carmi et al ${ }^{[9]}$ No such association was found in our study. Liver biopsy is important in cases where radiology has overlapping features.

Causes of Intrahepatic cholestasis other than EHBA and NH were PFIC, cholestatic liver disease with paucity of ductules and inflammatory biliary tract pathology. TORCH titres were raised in two cases probably explaining cholestasis. Presentation and laboratory parameters in cases of cholestasis are similar to EHBA and NH. Histologic features should be observed carefully for presence, absence or reduction of bile ductules. PFIC, especially type 3, can in fact have very similar histologic features to EHBA and sometimes even neonatal hepatitis. Elevated levels of GGT and free passage of dye into the intestine on HIDA scan favor a diagnosis of PFIC (Type 3). ${ }^{[10]}$

We diagnosed eight cases of cirrhosis on microscopy. Out of these, all cases were seen in children above one year of age. The commonest cause of cirrhosis in children is infectious disease followed by metabolic diseases ${ }^{[11]}$ None of the cases showed evidence of hepatitis B infection on histology. Jia-An-Zhu et al compared results of liver biopsy and USG in 28 children with liver cirrhosis concluding that USG showed abnormal characteristics which were not specific to the disease thereby reinforcing the necessity of USG guided liver biopsy in diagnosis of children with liver cirrhosis. ${ }^{[12]}$ However, at the stage of cirrhosis, it may not always be possible to identify the etiology even on liver biopsy.

Ishak scoring system should be used for grading and staging of chronic hepatitis ${ }^{[13]}$ None of cases in our study was positive for $\mathrm{HbS} \mathrm{Ag}$. The causes are variable and include chronic hepatitis $\mathrm{B}$ and $\mathrm{C}$ and autoimmune hepatitis. Serology is important for etiology. Liver biopsy helps in staging in these cases ${ }^{[5,14]}$

Autoimmune Hepatitis: (AIH)has broad clinical spectrum including asymptomatic individuals with abnormal laboratory results, clinical symptoms similar to those of acute viral hepatitis, and hepatic insufficiency or even cirrhosis $^{[}{ }^{[15,14]}$

The cases were diagnosed based on characteristic histologic features like interface hepatitis and dense infiltration of portal tracts by mononuclear cells and predominance of plasma cells. In a study by Jimenez-Riviera et al, of 200 cases, the median age range was 12 years for type $1 \mathrm{AIH}$ and 10 years for Type $2 \mathrm{AIH}$. One hundred and nine patients had anti-nuclear and/or smooth muscle antibody (ANA/SMA) titres positive and 18 patients had liver/kidney microsomal antibody (LKM-1) titres positive. ${ }^{[16]}$ Similar histologic findings were seen in the study by Dehghani et al. ${ }^{[17]}$ They studied 87 children of AIH. Mean age of presentation was 10 years with female predominance. Antinuclear, anti-smooth muscle, and anti LKM antibodies were positive in 14/62, $22 / 53$ and $6 / 40$ patients respectively. Twenty six patients were seronegative, and autoantibodies were not available 
in 19 cases. Seronegative AIH has typical appearance of AIH on histology, responds to immunosuppression, but lacks detectable autoantibodies ${ }^{[18]}$ This is a rare form of AIH in adults, but its prevalence and clinical characteristics remain to be defined in children.

Histologic features favored a tuberculosis etiology in all cases of granulomatous hepatitis. Other causes need to be ruled out like fungal or parasitic infection by using special stain for fungus such as GMS ${ }^{[5]}$ Monajemzadeh et al evaluated prevalence and etiology of granulomatous hepatitis in liver biopsies of 33 Iranian children. [19] Etiological agents identified were mainly mycobacterial infection followed by sarcoidosis, HCV infection and kala-azar suggesting that mycobacterial infection is an important cause of granulomatous hepatitis.

Wilson Disease: was found in three cases. Clinically, they presented with abdominal distension and neurological symptoms. Histologic features seen in our cases were cirrhosis with glycogenated nuclei and occasional focus of hepatocyte necrosis. The characteristic histologic features. may not seen in all cases. The findings on a liver biopsy are very variable and depend on the extent of liver damage. ${ }^{[5}$, ${ }^{20}{ }^{2}$ Hence a diagnosis of Wilson Disease can be confidently made only after correlation with serum ceruloplasmin, urinary copper levels and dry weight of copper. ${ }^{[20]}$ In our study, three cases were diagnosed cases of Wilson disease. Two out of these cases had high urine copper and low serum ceruloplasmin levels

PAS positivity and diastase sensitivity was noted in seven out of 11 cases of Glycogen storage disease which supports a diagnosis of Glycogen storage disease (all types except Type IV). Presence of diastase resistant PAS positivity favours a diagnosis of Glycogen storage disorder Type IV. Enzyme levels were not available in these cases. However, the histology was quite classical. Presence of distended hepatocytes due to glycogen obliterates the sinusoids and shows a mosaic pattern. Presence of glycogenated nuclei in glycogen storage disease differentiates it from carbohydrate metabolic disorder. Sub-typing of these disorders in difficult without enzyme studies. ${ }^{[5,14]}$

The wrinkled tissue paper appearance of the cytoplasm of distended Kupffer cells is diagnostic of Gaucher disease. PAS stain makes the wrinkles prominent and should be performed.

Secondary hemosiderosis was seen in cases of Thalassemia major. Liver biopsy is usually performed in neonates when neonatal (hereditary) hemochromatosis is suspected. ${ }^{[14]}$ It is usually not performed for secondary causes except to confirm fibrosis.

Hepatic tumors: Hepatoblastoma showed fetal histologic type which is the commonest sub type (Figure 2f). Alpha fetoprotein was high in this case. The fetal type of hepatoblatoma may resemble normal liver parenchyma. In such a case, correlation with serum AFP levels is very important as it is a sensitive marker for hepatoblastoma. We reported one rare case each of Non Hodgkins Lymphoma and Hepatic Sarcoma. There is a definite role of liver biopsy in diagnosing hepatic masses as radiology may be overlapping. ${ }^{[14]}$

To conclude, paediatric liver biopsy is of value in diagnosis of disorders where clinical and laboratory parameters overlap. At the same time, clinical and laboratory correlation is a must due to overlapping histologic features of many liver diseases. Thus, liver biopsy assisted by a good clinical examination, radiology and routine as well as specific laboratory evaluation can succeed in diagnosis and follow up of majority of liver diseases.

\section{References}

1. Ahmad M, Afzal S, Roshan E, et al. Usefulness of Needle Biopsy in the diagnosis of Paediatric Liver Disorders. J Pak Med Assoc 2005 ;55:24-8.

2. Marion AW, Baker A J, Dhawan A. Fatty liver disease in children. Arch Dis Child. 2004;89:648-52.

3. Muthuphei M N. Childhood liver diseases in Ga- Rankuwa hospital, South Africa. East Afr Med J 2000 ;77:508-509.

4. Chardot C. Biliary atresia. Orphanet Journal of Rare Diseases 2006 ;1:1-9.

5. Hicks J, Mani H, Stocker J T.The Liver, Gallbladder, and Biliary Tract. In: Husain AN,Stocker J T, Dehner L P, editors. Pediatric Pathology. 4th edition. Wolters Kluwer; 2015.640-742.

6. Kahn E. Biliary atresia revisited. Pediatr Dev Pathol. 2004 ;7:109-24.

7. Shah I, Bhatnagar S, Rangarajan V, Patankar N. Utility of Tc99m-Mebrofenin hepato-biliary scintigraphy (HIDA scan) for the diagnosis of biliary atresia. Trop Gastroenterol. 2012;33:62-4.

8. Torbenson M, Hart J, Westerhoff M, et al. Neonatal giant cell hepatitis: histological and etiological findings. Am J Surg Pathol. 2010;34:1498-503.

9. Gupta L, Bhatnagar V.A study of associated congenital anomalies with biliary atresia. J Indian Assoc Pediatr Surg. 2016;21:10-3

10. Srivastava A. Progressive Familial intrahepatic cholestasis. J Clin Exp Hepatol 2014;4:25-36 
11. Pinto RB, Schneider ACR, da Silveira TR. Cirrhosis in children and adolescents: An overview. World J Hepatol 2015;7:392-405.

12. Jia-An Zhu, Bing Hu. Ultrasonography in predicting and screening liver cirrhosis in children: A preliminary study. World J Gastroenterol 2003 ;9:2348-9.

13. Alvarez G, Wang H L. The Liver. In: Humphrey PA, Dehner L P, Pfeifer J D, editors. The Washington Manual of Surgical Pathology, first edition. Wolters Kluwer: Lippincott Wiliams and Wikins;2010. 210-11.

14. Tannapfel A, Dienes HP, Lohse AW: The indications for liver biopsy. Dtsch Arztebl Int 2012; 109: 477-83.

15. Mieli-Vergani G, Heller S, Jara $\mathrm{P}$, et al. Autoimmune Hepatitis. JPGN 2009;49:158-164.
16. Jiménez-Rivera C, Ling SC, Ahmed N. Incidence and Characteristics of Autoimmune Hepatitis. Pediatrics 2015;136:e1238-48

17. Dehghani S, Haghighat $\mathrm{M}$, Imanieh $\mathrm{M}$, et al. Autoimmune Hepatitis in Children: Experiences in a Tertiary Center. Iran J Pediatr 2013; 23:302-8.

18. Krawitt EL. Clinical features and management of autoimmune hepatitis. World J Gastroenterol. 2008;14 : 3301-5.

19. Monajemzadeh M, Haghi M, Najafi M, et al. Granulomatous hepatitits : A 10 year study in Iranian children. Research journal of biological sciences 2009;4:503-5.

20. Roberts EA, Schilsky ML. Diagnosis and Treatment of Wilson Disease: An Update. Hepatology 2008;47:2089-111.

*Corresponding author:

Dr.Pragati Aditya Sathe, A/7, Jeevan Sudha CHS, C D Barfiwala Road, Andheri West, Mumbai-400058,India.

Email: pragativk@yahoo.com

Date of Submission : 19.12.2016

Date of Acceptance : 10.04.2017

Financial or other Competing Interests: None.

Date of Publication : 31.08.2017 